\title{
La Industrialización del heno de alta calidad en Costa Rica.
}

\section{Origen de la demanda tecnológica}

En 1991, los investigadores del INTA empezaron a gestar la posibilidad de investigar y desarrollar tecnología para mejorar la calidad del heno disponible comercialmente en Costa Rica. Se sabía de la mala calidad de este heno, porque se producía en un $100 \%$, en condiciones de secano, en donde la edad de rebrote del forraje henificado, factor fundamental en la calidad de cualquier forraje, oscilaba de tres a siete meses. Como sería de esperar con estas bajas calidades, dicho forraje sólo podría ser utilizado, al igual que las pacas de arroz, para sostener o disminuir la pérdida de peso de los animales, durante épocas críticas de escasez de forrajes y no para la producción de leche o carne.

Esta inquietud resultó: en la incorporación, con fondos PROGASA, de un espectroscopio de reflejo de onda cercana al infrarrojo (NIRS=Near Infrared Reflectance Spectroscopy), al laboratorio de piensos y forrajes del INTA, el cual permite realizar análisis rápidos de nutrientes de los alimentos y forrajes y en la elaboración de un protocolo y ejecución de la actividad de investigación de 1992 a 1994.

En 1997, durante una visita de los miembros del Sector Agropecuario al Distrito de Riego Arenal Tempisque (DRAT), para analizar el Plan Estratégico que se ejecutaba en la zona, los investigadores del INTA propusieron el desarrollo de tecnología para la producción de heno de alta calidad en sistemas de producción bajo riego, como una opción productiva para las familias del DRAT y como una oportunidad para poner en el mercado, un forraje de mucho mayor calidad, para la producción animal.

\section{Consolidación del proyecto}

Establecida la necesidad tecnológica, en 1998 se elabora e inicia la ejecución del proyecto "Industrialización del heno de calidad en Costa Rica", cuyos enfoques básicos fueron agroambiente, agrocadena, género, diversificación e innovación.

\section{Fase 1. Estación Experimental Enrique Jiménez Núñez. 1998-2000.}

En esta estación experimental del INTA ubicada en Cañas, Guanacaste, se iniciaron las investigaciones bajo sistemas de riego con el pasto Transvala, por ser ésta la especie forrajera más utilizada en la producción de heno comercial. Para ésto se utilizaron parcelas pequeñas, sobre las cuales se midió el efecto del riego, la edad de rebrote y el nivel de fertilización nitrogenada, sobre la producción y la calidad del forraje, así como una estimación de la rentabilidad de las prácticas aplicadas. Esta fase experimental, demostró que era posible triplicar la calidad (12 vs. 4 \% proteína) y la producción del heno (1.400 vs. 500 pacas/ha/ciclo) con respecto al sistema de producción de secano y que la rentabilidad aproximaba los $\$ 400 \mathrm{mi} / \mathrm{ha}$ ( $\phi 395 / \$)$.

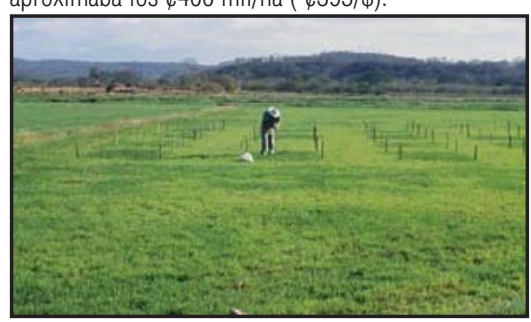

Fase 2. Validación de los resultados experimentales en el DRAT. 2000 - 2002.

Los resultados obtenidos en la estación experimental fueron validados en las parcelas de Floriberto Esquivel (alias Caja de Fósforo, 1950-2006 q.d.D.g.) primer productor de heno de alta calidad en Costa Rica y de Olidier Rojas, en el Asentamiento Campesino la Falconiana. Para ello se utilizaron tanto parcelas pequeñas, como áreas comerciales que fueron establecidas con este propósito. La producción y calidad fueron confirmadas totalmente mediante la validación, aplicando $65 \mathrm{~kg}$ de N por hectárea (tasa que no evidenció acumulación de N02 ni N03, a 50 cm en aguas de percolación), 45 a 50 días de rebrote $y$ de tres a cinco riegos durante cada corte, para un total de cuatro cortes por ciclo productivo (noviembre a mayo). La rentabilidad de $\$ 380 \mathrm{mil} / \mathrm{ha}$ por ciclo, fue menor, pero cercana a la obtenida en la estación experimental. Simultáneamente a este proceso de validación, se demostró el efecto del heno de calidad, sobre la ganancia de peso de toretes, comparado al del heno comercial de secano. También se muestrearon alrededor de la región, los diferentes henos comerciales disponibles, encontrándose contenidos de proteína entre 3,5 y $6 \%$ en heno de transvala de secano y de 3,5 a $4,5 \%$ en pacas de arroz, comparados con los contenidos de 8 a $12 \%$ en pacas de transvala producidas con la nueva tecnología. Producto de esta fase, más de 50 ha producen heno de muy buena calidad de transvala en el DRAT.

\section{Fase 3. Heno de leguminosa. 2002-2004.}

Aún cuando ya se contaba con la tecnología para producir heno de mucha mayor calidad que el heno comercial, se consideró que se podía mejorar aún más la calidad, para contribuir a la intensificación de los sistemas de producción animal. Para ello FUNDECOOPERACION financió el proyecto que investigaría y validaría, el desarrollo de una tecnología de heno de alta calidad, basada en el uso de leguminosas.

Después de probar diferentes alfalfas de norte y sur América, así como Clitoria ternatea, traída de México y un híbrido natural de Arachis sp. de tallo hueco existente en el país, se logró encontrar una mezcla entre pasto transvala y esta última leguminosa hibrida de Arachis, que mostró niveles de producción cercanos al transvala fertilizado bajo riego (1.200 vs 1.400 pacas por ha/ciclo de producción). Esta mezcla además, presentó contenidos de proteína de hasta 15 $\%$ y digestibilidades de hasta $65 \%$, jamás alcanzables con transvala puro, además de que el sistema no utiliza fertilización nitrogenada ya que la leguminosa incorpora el $\mathrm{N}$ naturalmente.

\section{Fase 4. Fomento de producción y mercado del heno de alta calidad. 2004-2006.}

Dada las calidades y producción obtenidas con este desarrollo tecnológico del INTA, se unieron todas las instituciones del sector agropecuario regional del DRAT, con el apoyo financiero de FUNDECOOPERACION nuevamente, para el desarrollo de la actividad comercial del nuevo producto. Además una consultoría en la fase anterior, demostró el potencial de desarrollar el mercado de heno de alta calidad.

Como resultado de esta última fase y de todo el proyecto en su conjunto, se constituyó la Organización de Productores Agroindustriales de Bagaces (APAIB), que agrupa a 25 agricultoras y agricultores. Ellos producen actualmente en 75 ha establecidas del cultivo asociado, 90 mil pacas por año de Nutriheno de APAIB, de alta calidad, que distribuyen en todo el país, con un ritmo creciente de demanda y producción. Simultáneamente se hicieron ensayos con toretes de engorde y ganado lechero especializado, demostrándose los beneficios biológicos y económicos, en la producción animal, utilizando el heno de alta calidad, Nutriheno de APAIB.

En el 2005 se liberó oficialmente, mediante los procesos establecidos por la Oficina Nacional de Semillas (ONS), el cultivar INTA-Falconiana (Arachis sp. CIAT 18744A) especie leguminosa base forrajera del Nutriheno. Se desarrolló también, la curva de calibración del NIRS que permite muestrear y analizar la calidad de lotes de heno, en tan sólo dos días.

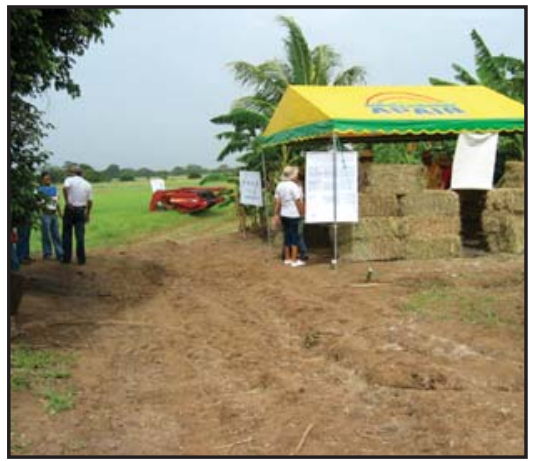

Ésto hizo posible registrar la marca hemeng y respaldarla con el etiquetado de su contenido nutricional. Se dieron los primeros pasos para desarrollar la comercialización del heno en subastas y así ir cambiando hacia una cultura de calidad del heno. Producto directo e indirecto del proyecto, unas 150 ha están produciendo heno de calidad superior en el DRAT. El proyecto en su totalidad tuvo un costo cercano a los $\$ 200$ millones, cuyos resultados generaron una actividad alrededor del heno de calidad en el DRAT, que a la fecha ha dinamizado la economía en la cadena del heno, hasta la producción de leche y carne, en cerca de $\$ 800$ millones. Esto demuestra que "la investigación impulsa el desarrollo de empresas exitosas". La información mediada para productores y técnicos, se ha transferido y está disponible en Plataforma PLATICAR del INTA, la científica inicia su publicación con el presente número de la Revista del INTA "Alcances Tecnológicos".

Dr. Jorge Morales, Investigador, INTA

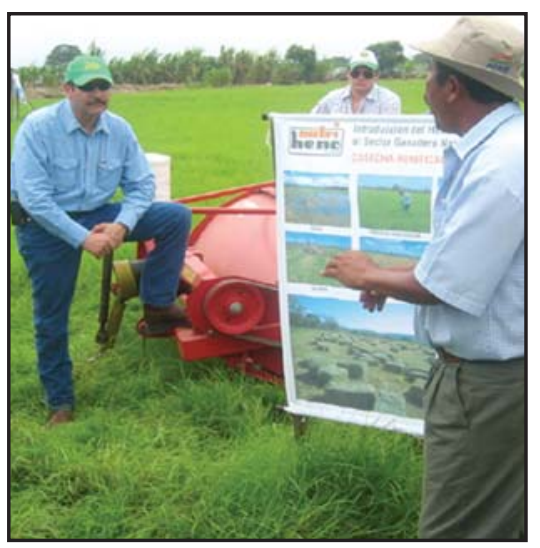

